\title{
ON AN APPLICATION OF VIETORIS'S INEQUALITY
}

\section{JANUSZ SoKÓŁAND PAWEŁ WiTOWICZ}

Abstract. The radius of starlikeness for polynomials with zeroes distributed at certain curves in the unit disc as well as the case in which zeroes are concentrated at a single point are considered and sharp bounds are obtained.

Mathematics subject classification (2010): Primary 30C45; Secondary 30C80.

Keywords and phrases: Univalent functions, starlike functions, Vietoris's inequality.

\section{REFERENCES}

[1] J. W. AleXAnder, Functions which map the interior of the unit disc upon simple regions, Ann. of Math. 17 (1915), 12-22.

[2] G. Brown, F. DAI, K. WANG, Extensions of Vietoris's inequalities I, Ramanujan J. 14 (2007), $471-$ 507.

[3] G. ENESTRÖM, Remarque sur un Théorème relatif aux racines d'équation $a_{n} x^{n}+\cdots+a_{0}=0$ où tous les coefficients soutréels et positifs, Tôhoku Math. J. 18 (1920), 34-36.

[4] A. Gluchoff, F. Hartmann, Starlikeness of polynomials and finite Blaschke products, Ann. Polon. Math., 93 (2008), 187-196.

[5] H. GRunsky, Zwei Bemerkungen zur konformen Abbildung, Jber. Deutsch. Math. Ver. 43 (1933), $140-143$.

[6] S. Koumandos, An extension of Vietoris's inequalities, Ramanujan J. 14 (2007), 1-38.

[7] R. Nevanlinna, Über die konforme Abbildund Sterngebieten, Oversikt av Finska-Vetenskaps Societen Forhandlingar 63 (A) 6 (1921), 48-403.

[8] L. VIETORIS, Über das Vorzeichen gewisser trigonometricher Summen, Sitzungsber, Öst. Acad. Wiss. Math.-Natur. Kl. S.-B. II 167 (1958), 125-135. 